\title{
Cost-effectiveness of cognitive-behavioral group therapy for dysfunctional fear of progression in chronic arthritis patients
}

\section{Mirjam Brach 1, Carla Sabariego 2,3,4, Peter Herschbach ${ }^{5}$, Petra Berg 5 , Ursula Engst-Hastreiter ${ }^{4}$, Gerold Stucki 1,3,4,6}

${ }^{1}$ Swiss Paraplegic Research, Postfach CH-6207, Nottwil, Switzerland

${ }^{2}$ Department of Physical Medicine and Rehabilitation, Ludwig-Maximilian University, Marchioninistr. 15, 81377 Munich, Germany

${ }^{3}$ ICF Research Branch of WHO FIC CC (DIMDI) at SPF, Nottwil, Switzerland

${ }^{4}$ Institute for Health and Rehabilitation Sciences, Ludwig Maximilian University, Marchioninistr. 17, 81377 Munich, Germany

${ }^{5}$ Department and Outpatient Clinic of Psychosomatic Medicine and Psychotherapy, Division of Psychosocial Oncology, Technical University of Munich, Ismaninger Str. 22, 81675 Munich, Germany

${ }^{6}$ Department of Health Sciences and Health Policy, University of Lucerne, Pilatusstrasse 20, 6000 Luzern 7, Switzerland

Address correspondence to Gerold Stucki, E-mail: gerold.stucki@paranet.ch

\begin{abstract}
Background Anxiety disorders are widespread in patients with chronic diseases such as rheumatoid arthritis (RA). This paper targets the costeffectiveness analysis of a cognitive-behavioral group therapy (CBT) in comparison to a client-centered, supportive-experiential group therapy (SET) in arthritis patients with dysfunctional fear of progression.

Methods From the societal perspective, direct costs were compared with the reduction of fear of progression over time. Means, their $95 \%$ confidence intervals $(95 \% \mathrm{Cl})$, the incremental cost-effectiveness graphic and the acceptability curve were obtained using 1000 nonparametric bootstrap replications.

Results A total of 174 RA patients were included in the economic evaluation. The estimated means (95\% CI) of direct costs and reduction of fear of progression were, respectively, $€ 7945.34(5075.59 ; 11335.08)$ and $0.25(-0.48 ; 0.99)$ for patients in the SET and $5619.25 €$

$(3950.67 ; 7708.52)$ and $0.94(0.29 ; 1.62)$ for patients in the CBT. As the majority of the cost-effect pairs after bootstrap analysis were located in the southeast quadrant of the cost-effectiveness plane, the CBT can be considered a dominant intervention.

Conclusion The main result of our study is the higher cost-effectiveness of CBT in comparison to SET in RA patients with dysfunctional fear of progression.
\end{abstract}

Keywords cognitive behavior therapy, cost-effectiveness, economics, health resource, rheumatoid arthritis

\section{Introduction}

Anxiety disorders are widespread in patients with chronic diseases such as rheumatoid arthritis (RA). ${ }^{1,2}$ In a recent study in Germany either a depressive or anxiety disorder was diagnosed in $24.3 \%$ of patients with inflammatory rheumatic diseases. ${ }^{3}$ In addition to the burden to patients and their families, fear and anxiety associated with medical conditions have important socio-economic consequences as high health care use costs and an important reduction of the work capacity. ${ }^{4-6}$ In Germany, high indirect annual costs of an average $\$ 11750$ were identified among RA patients. ${ }^{7}$ Consequently, interventions, which can reduce fear and anxiety of patients suffering from chronic conditions, would not only benefit patients directly, but could also contribute to the reduction of the economic burden to society.

To our knowledge, chronic arthritis patients with fear of progression undergoing inpatient rehabilitation in Germany receive no structured psychological therapy. However, fear

\footnotetext{
Mirjam Brach, Research Manager Carla Sabariego, Research Fellow, Psychologist

Peter Herschbach, Professor, Head of the Section of Psychosocial Oncology

Petra Berg, Head of Outpatient Psychological Clinic

Ursula Engst-Hastreiter, Clinical Psychologist

Gerold Stucki, Professor and Chairman
} 
of progression is potentially amendable to cognitivebehavioral therapies (CBT): a recent study by Herschbach found a statistically significant long-term reduction of fear of progression in cancer patients after CBT as well as after a client-centered, supportive-experiential group therapy (SET), but not among chronic arthritis patients. ${ }^{7,8}$ However, CBT for patients with generalized anxiety disorders is costeffective., Additionally, a recent study shows that RA patients had statistically significant lower health-care use after CBT. $^{10}$ It would be therefore important from a societal perspective to demonstrate whether CBT can also result in a reduction of direct medical and indirect costs in the RA patients with dysfunctional fear of progression. More specifically, a hypothesis can be formulated as to whether CBT can be more cost-effective than SET.

However, there is a lack of economical evaluations investigating the cost-effectiveness of psychotherapeutic interventions for RA patients with anxiety disorders. The objective of our study therefore was to perform an incremental cost-effectiveness analysis of the results of the randomized controlled trial (RCT) published by Herschbach et al. ${ }^{11}$ comparing a CBT focusing on fear of progression to client-centered SET.

\section{Materials and methods}

\section{Study design}

We performed an economic evaluation alongside an RCT. One hundred and seventy-four RA participants were recruited between November 2002 and December 2003 in one rehabilitation clinic in the region of Bavaria, Germany. Patients willing to participate were allocated to an intervention arm using external randomization and were blind with regard to group assignment. The inclusion criteria of the study were: RA, minimum age 18 years old, inpatient rehabilitation and increased fear of progression measured with the standardized fear of progression questionnaire. ${ }^{12}$ Patients were excluded for having cognitive impairment, being bedridden or poor command of the German language. Patients were assessed at baseline, post-intervention and at the 3- and 12-month follow-up after discharge. ${ }^{11}$

\section{Interventions}

All patients underwent the same 3-week inpatient rehabilitation program. In addition to the standard rehabilitation program, patients in both CBT and SET groups received four sessions of group psychotherapy, each lasting $90 \mathrm{~min}$. The sessions were led by trained psychotherapists and all sessions were supervised. Both CBT and SET were manualized with regard to structure but content (topics and intervention) was predefined solely in CBT. While the CBT was a directive and specific intervention aimed at confronting patients with their fears and at learning to cope with them, the SET was a client-centered, supportive-experiential group therapy intervention based on the work of Carl Roggers. ${ }^{11}$ The SET was a non-directive and unspecific intervention focusing on emotional expression, mutual support and reassurance, and social comparison. ${ }^{11}$

\section{Health-care utilization and costs}

The economic evaluation was performed from the societal perspective. As this study was conducted over a 1 year period, costs and effects were left undiscounted. Incremental interventions costs were calculated based on the duration of the therapy modules, group size and the minute salaries of psychologists. Following Guidelines of the Working Group Methods in Health Economic Evaluation (AG MEA), ${ }^{13-15}$ a $20 \%$ charge was added to the calculated costs because of the time spent learning the manuals, preparing the sessions and conducting supervisions.

Patients of SET and CBT completed a retrospective health-care resource use assessment questionnaire at baseline and 12 months after rehabilitation. The questionnaire was developed following the guidelines proposed by the AG MEA. ${ }^{13-15}$ Resource use volumes were combined with unit costs to obtain a net cost per patient over the entire period. If resource use was obtained for recall periods shorter than 12 months, resource use data were extrapolated to obtain annual figures. Direct medical costs included visits to practitioners, non-physician service utilization, hospital treatments, day care, medication, devices and aids. Direct non-medical costs included leisure time loss of patients due to participation on self-help groups and of parents or friends due to the voluntary assistance of the patient. Indirect costs were assessed using the human capital approach and were calculated based on sick leave days. All costs were calculated in euros.

\section{Effectiveness data}

We report means and standard deviations of the fear of progression score and of the mental scale of the SF12 estimated in the original RCT.

\section{Cost-effectiveness analysis}

We performed an incremental cost-effectiveness analysis (CEA) comparing both intervention groups (CBT and SET) and calculated the incremental cost-effectiveness ratio 
(ICER), i.e. the difference in average costs divided by the difference in average effects between these two groups. Indirect costs were not included in the CEA because of $51.15 \%$ missing values with regard to sick leave in the follow-up. Costs were the mean direct costs (medical and non-medical) within the first year after discharge. Effectiveness was measured with the standardized fear of progression questionnaire, which comprises 43 items relating to 5 dimensions: affective reactions, partnership/family, occupation, loss of autonomy and coping with anxiety. We used as an effectiveness measure of the total score, i.e. the sum of the subscales' mean scores excluding the coping scale. This score has a range of values between 4 (minimum) and 20 (maximum). ${ }^{12,16}$ The mean fear of progression at the 12-month follow-up minus the mean fear of progression at baseline was used as an effectiveness measure. As a reduction of the fear of progression was aimed, effectiveness is expected to have negative figures. However, negative effectiveness measures in CEA are interpreted as being less effective. Owing to this fact we multiplied the effectiveness regarding the outcome fear of progression by negative 1 in order to get positive figures in the event of more effects.

In order to increase the comparability of this study to the literature, we also performed an incremental CEA using the mental score of the questionnaire for general health status (SF12) at the 12-month follow-up as the effect outcome. ${ }^{17}$ The mental score of the SF12 was chosen for comparison because of its close association with the Fear of Progression Questionnaire. $^{12}$

\section{Unit costs}

All costs were calculated for the year 2004 (Table 1).

Medication was valuated on the basis of prices of the online German drugs index book. ${ }^{18}$ Devices and aids were valuated with prices charged by the health insurance society of Bavaria. ${ }^{19}$ Day-care treatment was purchased with prices charged in 2004 at the University of Munich Hospital. All other index costs were proposed by the AG MEA. ${ }^{13-15}$ The proposed index costs were originally estimated for the year 2001. We extrapolated the unit costs for 2004 using a factor of 0.025 for the first year and 0.020 for the following years as suggested by the AG MEA.

\section{Statistical analysis}

We used non-parametric bootstrap techniques with 1000 replications to estimate mean values and 95\% confidence intervals $(95 \% \mathrm{CI})$. In order to compare the means of the groups, we calculated the achieved significance level (ASL)
Table 1 Prices applied in the economic evaluation

\begin{tabular}{|c|c|c|c|}
\hline Cost categories & $\begin{array}{l}\text { Price } \\
(€)\end{array}$ & Cost categories & Price $(€)$ \\
\hline Physician visits ${ }^{a}$ & & Daycare treatment & \\
\hline General practitioner & 16.03 & Internal medicine & 252.00 \\
\hline Neurologist & 23.02 & Surgical & 484.00 \\
\hline Internist & 34.62 & Gynecology & 175.00 \\
\hline Orthopedist & 23.43 & Neurology & 269.00 \\
\hline \multirow[t]{4}{*}{ Rheumatologist } & 30.18 & Radiology & 235.00 \\
\hline & & Physical medicine & 270.00 \\
\hline & & Oncology & 575.00 \\
\hline & & Pain unit & 285.00 \\
\hline Inpatient treatment ${ }^{\mathrm{b}}$ & & $\begin{array}{l}\text { Outpatient treatment } \\
\text { (hospital) }\end{array}$ & \\
\hline Internal medicine & 310.62 & Gastroenterology & 23.02 \\
\hline Surgical & 349.55 & Gynecology & 26.88 \\
\hline Gynecology & 377.33 & Surgical & 22.94 \\
\hline Gastroenterology & 358.45 & Urology & 28.88 \\
\hline Urology & 374.03 & Dermatology & 15.63 \\
\hline Dermatology & 332.87 & Internal medicine & 34.62 \\
\hline Neurology & 357.34 & Orthopedic & 23.43 \\
\hline Orthopedic & 275.02 & Cardiology & 61.79 \\
\hline Cardiology & 348.44 & Radiology & 83.54 \\
\hline Radiology & 416.30 & Pneumology & 37.82 \\
\hline Rehabilitation oncology & 105.00 & Rheumatology & 30.18 \\
\hline Rehabilitation orthopedic & 102.00 & Psychology & 47.51 \\
\hline Mean value & 390.00 & Mean value & 23.02 \\
\hline Psychotherapyc & 47.51 & $\begin{array}{l}\text { Self-help groups } \\
\text { (patient hour price) }\end{array}$ & 17.80 \\
\hline Physiotherapy & 21.89 & $\begin{array}{l}\text { Time of relatives } \\
\text { (hour price) }\end{array}$ & 17.80 \\
\hline Massage & 9.70 & Sick leave days ${ }^{d}$ & 89.00 \\
\hline Ergotherapy & 29.00 & Psychologist minute $\mathrm{e}^{\mathrm{e}}$ & 0.4910 \\
\hline
\end{tabular}

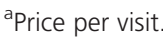

${ }^{\text {b}}$ Price per day.

'Price per session.

dPrice of a calendar day.

eSalary of a rehabilitation clinic staff.
}

using bootstrap- $t$ methods. The achieved significance level corresponds to the $P$-value of a $t$-test and will be called pboot here. ${ }^{20}$ Because of multiple testing a probability $P$-value of less than 0.005 with Bonferroni correction (0.05/9) was considered significant. The uncertainty surrounding the estimates of the cost-effectiveness was illustrated by a costeffectiveness acceptability curve (CEAC). The threshold value payers are willing to pay for a new intervention is usually not known. Therefore, in the CEAC probabilities of superior cost-effectiveness of CBT compared with SET are calculated for an arbitrary range of potential threshold 
values that decision-makers could be willing to pay for the implementation of CBT. The mean costs and mean effects distributions obtained from the 1000 replications were used to plot the incremental cost-effectiveness graphic and the CEAC. As the distribution of the bootstrap estimates of costs and effects was symmetric, we use the percentile method to calculate $\mathrm{CI}^{21}$

Uncertainty in the cost and effectiveness estimates can be allowed by sensitivity analysis, statistical inference or some combination of the two approaches. ${ }^{22}$ We addressed the uncertainty presenting CI obtained from bootstrap samples and estimating acceptability curves for the ICERs. ${ }^{23}$ All statistical calculations inclusive of the bootstrap procedure, the estimation of the incremental cost-effectiveness graphics and the CEAC were performed using SPSS version 14.0.

\section{Results}

\section{Study population}

Health resource use data were collected for 174 patients randomized either in the CBT $(n=87)$ or in the SET $(n=87)$ group. The average age of patients in the SET and in the CBT groups was 47.45 (SD 8.91) and 45.43 (SD 9.75), respectively. The percentage of male participants was $23 \%$ in the SET and 31\% in the CBT group. At baseline, $79.3 \%$ of SET and $75.8 \%$ of CBT participants were employed. Complete indirect cost data of at baseline employed patients were available at the 12-month follow-up for 89 patients (37 SET; 52 CBT). As described elsewhere, patients of both groups were comparable to sociodemographic characteristics, disease severity and comorbidities. ${ }^{24}$

\section{Dropouts}

By the 12-month follow-up 55 patients (34 SET and 21 CBT) had dropped out: $18.2 \%$ did not want to participate anymore, 9.1\% could no be contacted anymore, $72.8 \%$ did not return the case record form. Drop outs were very similar to participants considering age, sex and employment status. Participants and drop outs incurred similar direct medical costs at baseline but drop outs on average incurred higher indirect costs [€6108.21 (95\% CI: 3484.54; 8731.87)] than participants [€4214.41 (95\% CI: 2894.88; 5533.93)].

\section{Health-care utilization and costs}

Both CBT and SET were performed as an additional therapy module to a standard 3-week inpatient rehabilitation program. The incremental cost of adding either CBT or SET to a standard 3-week inpatient rehabilitation program was about $€ 47$ per patient or $€ 282$ per group.

Patients of the CBT at the 12-month follow-up had fewer internist visits, fewer inpatient days, fewer day-care treatments, used fewer aids and devices, lower medication costs, fewer sick leave days and required less care giving from friends/relatives than patients of the SET. CBT subjects on average had at the 12-month follow-up fewer direct medical, direct non-medical and indirect costs as subjects who received SET but these differences were non-significant $(P=0.237, \quad P=0.381$ and $P=0.350, \quad$ respectively) (Table 2). Non-significant pre-post cost differences could be found in both groups. However, direct medical costs increased in both groups, mainly due to a substantial increase of medication costs. Patients of the SET also had more inpatient days, day-care treatment days and psychotherapies sessions in the follow-up.

\section{Effectiveness data}

The fear of progression mean score was at baseline 12.4 (2.6) in the CBT and 12.5 (2.5) in the SET group. At the 12-month follow-up, the fear of progression mean score was 11.4 (2.8) in the CBT and 11.8 (2.7) in the SET group. Regarding the mental score of the SF12, the mean scores at the baseline were 39.8 (10.9) and 38.5 (9.3) and at the 12-month follow-up 41.6 (9.4) and 40.2 (9.3) in the CBT and SET groups, respectively.

Table 2 Mean (95\% Cl) costs (in the last 12 months) at baseline and at the 12-month follow-up

\begin{tabular}{llll} 
Direct medical costs & Baseline & 12-month follow-up & P-value \\
\hline SET $(n=87)$ & $4562.93(3199.86 ; 6327.93)$ & $6205.07(3712.74 ; 9264.95)$ & $\mathrm{pboot}=0.532$ \\
CBT $(n=87)$ & $3176.61(2684.76 ; 3730.42)$ & $4324.86(2928.20 ; 6005.44)$ & $\mathrm{pboot}=0.183$ \\
SET $(n=87)$ & $1801.61(956.62 ; 3138.96)$ & $1748.43(901.30 ; 2883.40)$ & $\mathrm{pboot}=0.424$ \\
CBT $(n=87)$ & $1612.86(1067.10 ; 2262.52)$ & $1212.16(644.25 ; 1946.77)$ & $\mathrm{pboot}=0.636$ \\
SET $(n=72)$ & $5120.58(3457.38 ; 6895.82)$ & $6562.07(2871.20 ; 10650.62)$ & $\mathrm{pboot}=0.200$ \\
CBT $(n=76)$ & $4392.97(3042.55 ; 5936.39)$ & $4526.00(2395.13 ; 7017.97)$ & $\mathrm{pboot}=0.538$
\end{tabular}

Means and $\mathrm{Cl}$ calculated with 1000 bootstrap replications. Costs are presented in euros. 


\section{Cost-effectiveness analysis}

Both direct costs and reduction of fear of progression data were available for 100 patients. The estimated means (95\% CI) of direct costs and reduction of fear of progression were, respectively, $€ 7945.34(5075.59 ; 11335.08)$ and 0.25 $(-0.48 ; 0.99)$ in SET and $€ 5619.25$ (3950.67; 7708.52) and $0.94(0.29 ; 1.62)$ in CBT. Figure 1 shows the costeffectiveness plane with the $(95 \% \mathrm{CI})$ of the ICER. The difference $(95 \% \mathrm{CI})$ in effects and direct costs amounted to $0.69(-0.21 ; 1.67)$ and to $-€ 2326.09$ (-6167.26; 1047.12), respectively. CBT is therefore from a societal perspective a dominant intervention (less costly, more effective), with the resulting ICER (95\% CI) amounting to minus $€ 3371.14(-36533.54 ; 25916.96)$ for an additional unit of effect. Given the acceptability curve there is a $90.2 \%$ chance that CBT, compared with the SET, is cost-effective without a need for additional costs to payers, i.e. there is a probability of $90.2 \%$ that the ICER is negative, meaning that CBT is less costly and more effective (Fig. 2).

Both direct costs and quality of life data were available for 44 patients of the SET and 55 patients of the CBT. The estimated means $(95 \% \mathrm{CI})$ of all direct costs and of the SF12 mental scale were $€ 8711.40(5841.80 ; 12292.26)$ and 39.34 (36.42; 42.20) in SET and €5619.25 (3950.67; 7708.52) and 42.57 (40.11; 45.35) in CBT, resulting into a negative ICER (95\% CI) amounting to minus €960.29 (-6991.71;
2274.18) for an additional unit of effect. The majority of the cost-effect pairs after bootstrap analysis were located in the southeast quadrant (plane not shown), suggesting that CBT is a dominant alternative. Given the acceptability curve (figure not shown) there is a $94.8 \%$ chance that CBT, compared with SET, is cost-effective without the need for additional costs to payers. As the close association between the Fear of Progression Questionnaire and the SF-12 also suggests a strong impact of fear of progression on overall quality of life, the similar results are not surprising. ${ }^{12}$

\section{Discussion}

\section{Main finding of this study}

In a recent RCT aiming to treat RA patients with elevated levels of fear of progression, no statistic significant longterm effects of CBT or SET could be shown. ${ }^{24}$ Our objective was to perform an incremental cost-effectiveness analysis of the results of this RCT published by Herschbach. The main result of our study is the higher cost-effectiveness of CBT in comparison to SET in RA patients.

\section{What is already known on this topic}

Currently, arthritis patients with dysfunctional fear of progression undergoing inpatient rehabilitation in Germany receive no psychological group therapy targeting FoP.

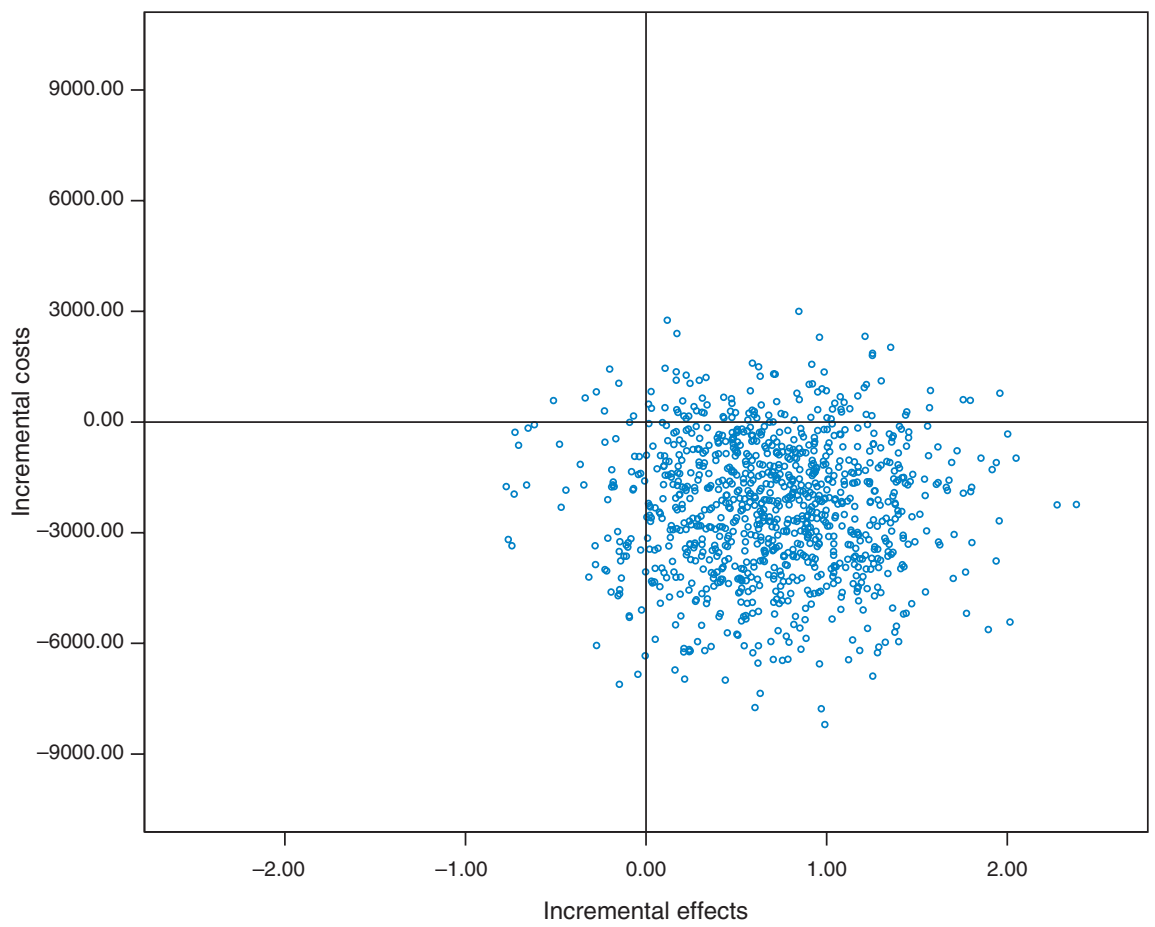

Figure 1 Incremental cost-effectiveness plane. Costs are direct costs in euros (medical and non-medical) within the first year after discharge. Effects are the reduction of fear of progression within the first year after discharge. Cost and effect pairs were estimated with 1000 bootstrap replications. 


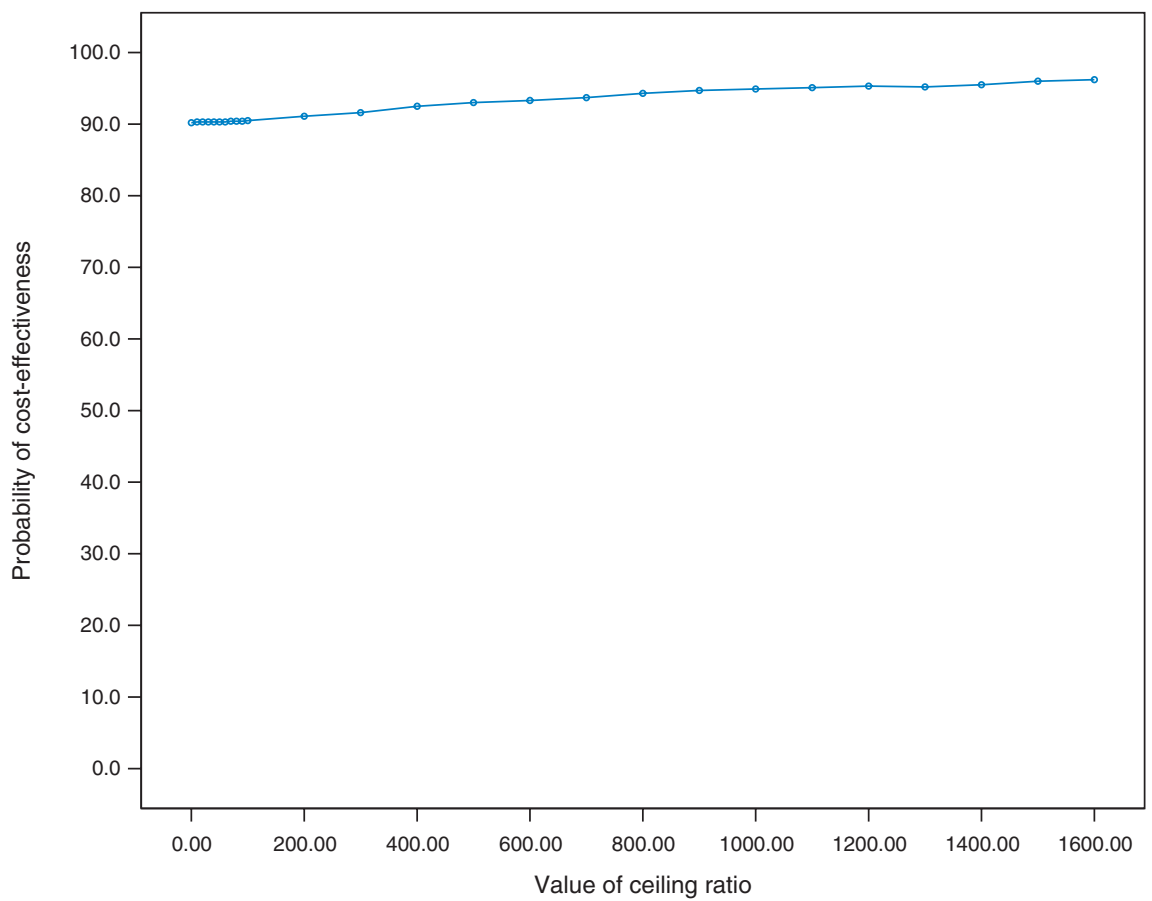

Figure 2 Acceptability curve regarding the probability of superior cost-effectiveness of CBT in comparison to SET. Curve estimated with 1000 bootstrap replications. The $x$-axis (values of ceiling ratio) represent a range of maximum monetary values in euros that a decision maker might be willing to pay for an additional unit of effect.

Although our CBT subjects had in the follow-up fewer direct and indirect costs as subjects who received SET, we failed to find statistically significant between-group differences. Similar results have been reported in the literature. An RCT of the arthritis self-management program also found no statistically significant between-group differences in health-care utilization of patients as RA, osteoarthritis or fibromyalgia. ${ }^{25}$ Also Schweikert's recent study, which comes very close to us in design and intervention but focuses on back pain disorders, found no statistically significant differences between intervention and control group regarding direct costs. ${ }^{26}$

Although CBT might improve productivity, few studies report data on indirect costs. A recent review targeting the economical impact of CBT in the treatment of mental disorders identified 22 cost-effectiveness studies but only four reported productivity loss data. ${ }^{8}$ In our study, a trend towards fewer sick leave days and less indirect costs could be observed in CBT group. We suppose therefore a positive impact of the intervention on sick leave. However, due to many missing values in the follow-up, we are not able to make reliable statements. A similar study for patients with chronic low back pain also reported less indirect costs in the intervention group but the difference was in a similar way statistically non-significant. ${ }^{26}$

\section{What this study adds}

While the cost-effectiveness of CBT for patients with mental disorders was often addressed, few economic evaluations targeted the economic impact of CBT in patients with chronic physical diseases such as RA. Two economic evaluations targeting the cost-effectiveness of adding CBT to a rehabilitation program for patients with chronic back pain came to the conclusion, the addition of CBT does not lead to statistic significant differences in costs and effects. ${ }^{26,27}$ In contrast, our study clearly states the superior cost-effectiveness of CBT for RA patients. A possible explanation and important difference between these studies and ours is that we enrolled patients not only with RA but also with a diagnosed acute mental disorder, which is actually the goal of CBT.

Our estimated ratios are negative and the magnitude of negative cost-effectiveness ratios is not informative. For example, if an intervention generates $€ 1000$ saving for 10 units of effect and an ICER of $€ 100$, doubling the effectiveness generates an ICER of $€ 50$. This seems economically less attractive but in reality is a better outcome. ${ }^{28}$ Moreover, the judgment whether an intervention is considered cost-effective depends on the willingness of payers to reimburse additional costs for additional clinical benefits. For both reasons, we calculated an incremental 
cost-effectiveness acceptability curve, which represents the probability that CBT is cost-effective compared with SET for a range of maximum monetary values that a decision maker might be willing to pay for an additional unit of effect. $^{29,30}$ Our calculated probability of $90.2 \%$ of higher cost-effectiveness of CBT without the need for additional costs to payers clearly states the superiority of this intervention in comparison to SET. An even higher probability is achieved (94.8\%) considering quality of life as the effect.

We observed in our study an increase, albeit statistically non-significant, of direct medical costs in the follow-up in both groups. A possible explanation could be the primary goal of the intervention, i.e. the reduction of the fear of progression. This effect was expected to translate into economic benefits but a reduction of health services utilization was not directly addressed in the therapy. Indeed, it is traceable that psychotherapies aiming at improvement of patients' psychological state cannot be expected to reduce medical costs to the same degree as interventions that have cost savings as primary goal. ${ }^{31}$ Another possible explanation for the increased costs might be the progressive and debilitating disease course of RA, which requires intensive medical care.

\section{Limitations of this study}

When interpreting the results of our study it is important to recognize that we preferred to compare two interventions stead of comparing CBT with no intervention at all. One consequence of this approach was that it is much harder to demonstrate statistically significant superiority in costs and effects. The recent development of cost-effectiveness acceptability curves reminds us though that the absence of a statistical difference in either costs or effects does not necessarily mean that two treatments cannot be distinguished. ${ }^{32}$ Indeed, because of the usual low power of economic evaluations performed alongside randomized clinical trials, the focus of cost-effectiveness studies should still be on estimating cost-effectiveness, even when either cost or effect differences lack conventional statistical significance. ${ }^{33}$

We also recognize that fear of progression, the effect used in the CEA, is an unusual outcome and eventually difficult to interpret. However, it is an important outcome because of its clinical relevance to patients and clinicians. In order to increase the comparability of our work with the literature, we decided to estimate an additional ICER using a well recognized and easier to interpret measure: the mental scale of the SF-12. Another possibility would have been to estimate QALYs using the SF-12. However, the accuracy of utilities estimated from the SF-12 was recently considered unsatisfactory, specially for older and less healthy groups, and inaccurate values can easily bias the conclusion of a cost-utility-analysis (CUA). ${ }^{34}$ Additionally, necessary parameters of a representative sample of the German population are not available so far. Due to the fact that the results of a CUA would have been questionable, we decided to go for a CEA using the SF-12 to increase the comparability of our study with other studies.

Our study has some limitations. First, our economical evaluation was performed alongside the main trial and is probably underpowered for the cost-effectiveness analysis. This is one reason for using bootstrap estimations and costeffectiveness acceptability curves. Second, we used a selfreport retrospective standardized questionnaire to collect information about health-care resource and this kind of data source is susceptible to recall bias. Third, we extrapolated a part of the direct cost components to obtain 1 year figures conservatively assuming that resource use increases constantly.

Our main result is the higher cost-effectiveness of CBT in comparison to SET for our sample of RA patients with high levels of anxiety. However, the magnitude of the economic benefits when comparing CBT with no intervention requires further investigation. Moreover, potential cost savings after rehabilitative interventions seem to be more likely in indirect than in the direct costs. For this reason, further economic evaluations should also focus more closely on the impact of psychological interventions on productivity loss.

\section{Acknowledgements}

We thank the clinics and our colleagues for their contribution to this study: H. Faller, E. Trempa, A. Reusch, W. Igel, A. Maucher, K. Behets, J. Stepien, J. Lerch, B. Hoßner, B. Heldmann, J. Markel, C. Berthold, B. Süß, R. Schröck, L. Schmid, K. Zellmann, G. Rauthe and W. Miehle.

\section{Funding}

This study was supported by the German Federal Ministry of Education and Research (BMBF) and the German Pension Insurance Administration (VdR).

\section{References}

1 Sinclair VG, Wallston KA, Dwyer KA et al. Effects of a cognitivebehavioral intervention for women with rheumatoid arthritis. Res Nurs Health 1998;21(4):315-26. 
2 Patten SB, Williams JV, Wang J. Mental disorders in a population sample with musculoskeletal disorders. BMC Musculoskelet Disord 2006;7:37.

3 Lowe B, Willand L, Eich W et al. Psychiatric comorbidity and work disability in patients with inflammatory rheumatic diseases. Psychosom Med 2004;66(3):395-402.

4 Hoffman DL, Dukes EM, Wittchen HU. Human and economic burden of generalized anxiety disorder. Depress Anxiety 2008 25(1):72-90.

5 Andlin-Sobocki P, Wittchen HU. Cost of anxiety disorders in Europe. Eur J Neurol 2005;12(Suppl. 1):39-44.

6 Wittchen HU. Generalized anxiety disorder: prevalence, burden, and cost to society. Depress Anxiety 2002;16(4):162-71.

7 Merkesdal S, Ruof J, Schoffski $\mathrm{O}$ et al. Indirect medical costs in early rheumatoid arthritis: composition of and changes in indirect costs within the first three years of disease. Arthritis Rheum 2001; 44(3):528-34.

8 Myhr G, Payne K. Cost-effectiveness of cognitive-behavioural therapy for mental disorders: implications for public health care funding policy in Canada. Can J Psychiatry 2006;51(10):662-70.

9 Heuzenroeder L, Donnelly M, Haby MM et al. Cost-effectiveness of psychological and pharmacological interventions for generalized anxiety disorder and panic disorder. Aust N Z J Psychiatry 2004; 38(8):602-12.

10 Sharpe L, Allard S, Sensky T. Five-year followup of a cognitivebehavioral intervention for patients with recently-diagnosed rheumatoid arthritis: effects on health care utilization. Arthritis Rheum 2008;59(3):311-6.

11 Herschbach P, Berg P, Waadt S et al. Group psychotherapy of dysfunctional fear of progression in patients with chronic arthritis or cancer. Psychother Psychosom 79(1):31-8.

12 Herschbach P, Berg P, Dankert A et al. Fear of progression in chronic diseases-psychometric properties of the Fear of Progression Questionnaire. J Psychosom Res 2005;58(6):505-11.

13 Burchert H, Hansmeier T, Hessel F et al. DRV-Schriften Band 16 Förderschwerpunkt Rehabilitationswissenschaften'-Ökonomische Evaluation in der Rehabilitation. Teil 2: Bewertung der Ressourcenverbräuche, Vol. 16. Frankfurt am Main: Verband Deutscher Renteversicherungsträger, 1999

14 Hessel F, Kohlmann T, Krauth C et al. DRV-Schriften Band 16 Förderschwerpunkt Rehabilitationswissenschaften' - Ökonomische Evaluation in der Rehabilitation. Teil 1: Prinzipien und Empfeblungen für die Leistungserfassung. Vol. 16. Frankfurt am Main: Verband Deutscher Renteversicherungsträger, 1999.

15 Krauth C, Hessel F, Hansmeier T et al. [Empirical standard costs for health economic evaluation in Germany - a proposal by the working group methods in health economic evaluation] Gesundheitswesen 2005;67(10):736-46.

16 Mehnert A, Herschbach P, Berg P et al. [Fear of progression in breast cancer patients-validation of the short form of the Fear of Progression Questionnaire (FoP-Q-SF)]. Z Psychosom Med Psychother 2006;52(3):274-88.
17 Gandek B, Ware JE, Aaronson NK et al. Cross-validation of item selection and scoring for the SF-12 Health Survey in nine countries: results from the IQOLA Project. International Quality of Life Assessment. J Clin Epidemiol 1998;51(11):1171-8.

18 Rote-Liste. Rote Liste Service GmbH. ECV Verlag, 2004.

19 AOK. Vergütungsliste für Orthopädische Hilfsmittel der Primärkassen in Bayern, 2004.

20 Barber JA, Thompson SG. Analysis of cost data in randomized trials: an application of the non-parametric bootstrap. Stat Med 2000;19(23):3219-36.

21 Carpenter J, Bithell J. Bootstrap confidence intervals: when, which, what? A practical guide for medical statisticians. Stat Med 2000;19(9):1141-64.

22 Drummond MF, O'Brien B, Stoddart GL et al. Methods for the Economic Evaluation of Health Care Programmes, 2nd edn. New York: Oxford Medical Publications, 1997.

23 Briggs AH. Statistical approaches to handling uncertainty in health economic evaluation. Eur J Gastroenterol Hepatol 2004;16(6):551 -61.

24 Herschbach P, Berg P, Waadt S et al. Group psychotherapy of dysfunctional fear of progression in patients with chronic arthritis and cancer. Psychother Psychosom, 2010;79(1):31-8.

25 Solomon DH, Warsi A, Brown-Stevenson $\mathrm{T}$ et al. Does selfmanagement education benefit all populations with arthritis? A randomized controlled trial in a primary care physician network. J Rheumatol 2002;29(2):362-8.

26 Schweikert B, Jacobi E, Seitz R et al. Effectiveness and costeffectiveness of adding a cognitive behavioral treatment to the rehabilitation of chronic low back pain. J Rheumatol 2006;33(12):2519-26.

27 Goossens ME, Rutten-Van Molken MP, Kole-Snijders AM et al. Health economic assessment of behavioural rehabilitation in chronic low back pain: a randomised clinical trial. Health Econ 1998;7(1):39-51.

28 Plant PK, Owen JL, Parrott S et al. Cost effectiveness of ward based non-invasive ventilation for acute exacerbations of chronic obstructive pulmonary disease: economic analysis of randomised controlled trial. BMJ 2003;326(7396):956.

29 Briggs A, Fenn P. Confidence intervals or surfaces? Uncertainty on the cost-effectiveness plane. Health Econ 1998;7(8):723-40.

30 Fenwick E, O’Brien BJ, Briggs A. Cost-effectiveness acceptability curves-facts, fallacies and frequently asked questions. Health Econ 2004;13(5):405-15.

31 Chiles JA, Lambert, Michael J et al. The Impact of psychological interventions on medical cost offset: a meta-analytic review. Clin Psychol Sci Pract 1999;6(N2):204-20.

32 Knapp M, Thorgrimsen L, Patel A et al. Cognitive stimulation therapy for people with dementia: cost-effectiveness analysis. $\mathrm{Br} \mathrm{J}$ Psychiatry 2006;188:574-80.

33 Briggs A. Economic evaluation and clinical trials: size matters. BMJ 2000;321(7273):1362-3.

34 Chuang LH, Kind P. Converting the SF-12 into the EQ-5D: an empirical comparison of methodologies. Pharmacoeconomics 2009;27(6):491-505. 